\title{
Partial cystectomy for muscle-invasive bladder cancer: a review of the literature
}

\author{
Taylor C. Peak, Ashok Hemal \\ Department of Urology, Wake Forest Baptist Health, Winston-Salem, NC, USA \\ Contributions: (I) Conception and design: All authors; (II) Administrative support: A Hemal; (III) Provision of study materials or patients: All authors; \\ (IV) Collection and assembly of data: TC Peak; (V) Data analysis and interpretation: TC Peak; (VI) Manuscript writing: All authors; (VII) Final \\ approval of manuscript: All authors. \\ Correspondence to: Ashok Hemal, MD. Department of Urology, Wake Forest Baptist Health, 1 Medical Center Blvd., Winston-Salem, NC 27157, \\ USA. Email: ahemal@wakehealth.edu.
}

\begin{abstract}
The radical cystectomy (RC) for muscle-invasive bladder cancer is one of the most morbid and complex urologic procedures performed today. To avoid these complications, the partial cystectomy (PC) has been offered as an alternative in carefully selected patients as a means of achieving equal oncologic efficacy with less morbidity. Selection criteria should include solitary tumors without concomitant carcinoma in situ (CIS) and amenable to resection with 1-2 cm margins in a normally functioning bladder. In addition to the standard work-up, random bladder and prostatic biopsies may be performed. The PC can be performed through an open, laparoscopic, or robot-assisted approach, each with acceptable outcomes. A number of techniques have been developed to identify and resect the tumor completely with negative margins, while preventing tumor spillage within the abdomen. While there are no randomized trials, single institution series have demonstrated acceptable oncologic outcomes in appropriately selected patients. Therefore, offering $\mathrm{PC}$ in the appropriate candidate, including those patients who do not accept or are unfit for the associated morbidity of a RC, represents an acceptable alternative.
\end{abstract}

Keywords: Urothelial carcinoma; partial cystectomy (PC); bladder preservation therapy; radical cystectomy (RC)

Submitted Oct 01, 2019. Accepted for publication Feb 17, 2020.

doi: $10.21037 /$ tau.2020.03.04

View this article at: http://dx.doi.org/10.21037/tau.2020.03.04

\section{Introduction}

There will be approximately 80,470 new bladder cancer cases and 17,670 related deaths in 2019, making it the most expensive and morbid of the urologic malignancies to treat (1). For patients who have disease invading the muscle-wall of the bladder, radical cystectomy (RC) is the gold standard of care. However, the RC is one of the most morbid and complex urologic procedures performed today. Even from the most experienced centers, complication rates are reported as high as $58-77 \%$ within the first 30 days, with readmission rates in $27 \%$ of patients (2-5). To avoid these complications, the partial cystectomy (PC) with bilateral pelvic lymph node dissection has been offered as an alternative in carefully selected patients. Data suggests that between $5 \%$ and $10 \%$ of patients meet certain selection criteria for undergoing a PC (6). Interestingly, despite comprising $7-10 \%$ of all cystectomies performed within the United States, only 2.8\% were performed for muscle invasive disease $(7,8)$. This article will review the body of literature regarding patient selection, surgical technique, and oncologic outcomes for the PC.

\section{Selection of surgical candidates}

Selection of the appropriate surgical candidate is critical to the oncologic success of a PC. Early series exploring the role of PC in muscle invasive bladder cancer were marked with high recurrence rates and poor oncologic control. However, more recent series from Memorial Sloan Kettering Cancer Center (MSKCC) and MD Anderson developed more strict criteria, including solitary tumor that would allow for 2 
$\mathrm{cm}$ margins, no carcinoma in situ (CIS), and no history of bladder tumors in other locations $(6,9)$. Highlighting the importance of selection, the MSKCC study found that $33 \%$ of patients with CIS had a local recurrence, versus $9 \%$ for those without. Fifty-six percent of multifocal tumors had a recurrence versus $8 \%$ with solitary tumor. They found that tumor location was not a predictor of recurrence or survival, although they did note that tumors along the posterior wall trended towards worse outcomes.

MD Anderson, likewise, reported on their experience, but used more strict criteria in offering PC, specifically in those patients with solitary tumor without concomitant CIS and amenable to resection with $2 \mathrm{~cm}$ margins and without need for ureteral reimplantation in a normally functioning bladder. Urachal tumors were excluded. They also performed random bladder biopsies in $58 \%$ of patients and prostatic urethral biopsies in $31 \%$ of men. They found that with these more strict selection criteria, there was a recurrence rate of $41 \%$.

In selecting patients, some groups recommend a restaging TURBT with random bladder and prostatic urethral biopsies to rule out concomitant CIS (10). Furthermore, an examination under anesthesia should be performed to ensure that there is no palpable mass, which would indicate clinical stage T3b and represent a relative contraindication to PC. Patients with significant voiding dysfunction or a small capacity bladder would also be inappropriate candidates for surgery. One group suggested grade should be considered when offering surgery, specifically those patients with high-grade disease (11). However, as our understanding of bladder cancer has advanced, it is now well understood that tumors that progress to muscle-invasion are by nature high grade.

Variant histology should also be considered in patient selection. There is no data specifically analyzing those patients with variant histology undergoing a PC. Certainly, for patients with histologic evidence of micropapillary, plasmacytoid, and sarcomatoid differentiation, an RC is the treatment of choice. These are known to be particularly aggressive, and as such PC would be contraindicated. While considered less aggressive relative to the variants mentioned above, squamous or adenocarcinoma differentiation may still be more likely to progress in comparison to those tumors with pure urothelial carcinoma. As such, it is uncertain whether a PC would provide adequate oncologic outcomes.

Bladder tumors within diverticula also represent a clinical situation in which a PC may be considered. The incidence of such tumors ranges from $0.8 \%$ to $14.3 \%$, with the most common histologic type being urothelial carcinoma $(12,13)$.
As there is no muscle in a bladder diverticula, these tumors may pose a greater risk of progressing to extravesical disease. And while transurethral resection may be feasible in some non-invasive tumors, $\mathrm{PC}$ represents a good alternative.

Urachal adenocarcinoma is also amenable to a PC. These tumors occur in less than $1 \%$ of all bladder cancer and $10 \%$ of all primary bladder adenocarcinomas (14). As such there are only single institution series and case reports within the available literature to suggest how to diagnose and treat these patients appropriately. However, in general, because by definition a urachal adenocarcinoma has to occur at the dome of the bladder, most patients are surgical candidates.

\section{Preoperative work-up}

All patient should undergo the standard work-up for muscleinvasive bladder cancer, just as they would for a RC. This includes labs (CMP and CBC), cross-sectional imaging of the abdomen and pelvis with intravenous contrast, as well as chest imaging with either a CT or chest X-ray. If there is concern for bone metastasis then a bone scan should also be performed. Random bladder biopsies may also be performed priory to surgery to exclude multifocal disease and/or CIS.

Special consideration should be taken in the diagnosis and management of urachal tumors as it can be difficult to distinguish between urachal and non-urachal bladder adenocarcinomas. While various criteria have been established to aid in this differentiation, a practical approach was developed by MD Anderson. The clinicopathologic features required for a diagnosis include an enteric-type adenocarcinoma in the midline of the bladder, location within the bladder wall, sharp demarcation between tumor and normal urothelium, and exclusion of a primary adenocarcinoma located elsewhere that has spread secondarily to the bladder (15). Urachal cancers may express detectable serum levels of carcinoembryonic antigen (CEA), CA125, and cancer antigen (CA) 19-9, especially in the setting of peritoneal carcinomatosis $(16,17)$. These markers should be drawn and may be followed for monitoring of disease response.

\section{Operative technique}

Prior to surgery, the surgeon should discuss the possibility that intraoperative findings may necessitate abandoning the PC, in favor of proceeding with an RC. The surgeon must also reiterate the fact that $\mathrm{RC}$ remains the gold standard, and that long-term oncologic outcomes may not be equivalent even in a carefully selected patient. The 
following discussion on operative technique will highlight the major steps involved in the surgery, with a focus on open, robotic, and laparoscopic approaches.

\section{Open PC}

The surgical technique for an open PC has been well described in the literature and begins by placing the patient in the supine position with small degree of flexion. A midline incision is made from the pubic symphysis to the level of the umbilicus. Dissection is carried down through the fascia, after which the peritoneum is opened and space of Retzius developed. If further exposure is required, then the vascular pedicle can be divided on one side. The colon is mobilized on each side along the white line of Told to expose the retroperitoneum. The ureters are identified and swept medially. A retractor of the surgeon's choice is then placed. At this point, a bilateral pelvic lymph node dissection should then be performed, with the same limits as a RC. This includes the aortic bifurcation proximally, Cooper's ligament distally, the genitofemoral nerve laterally, and the bladder medially. After the lymph node dissection, the bladder tumor is then resected. The bladder is opened away from the site of the tumor, preferably on the anterior bladder wall. The tumor is identified intravesically and then excised with a 1 to $2 \mathrm{~cm}$ margin. Alternatively, a flexible cystoscope can be inserted to identify the location of the tumor, and the cystotomy can be made over the light of the cystoscope. Specimens should be sent for frozen section to confirm negative margins. Next, the bladder is closed in two layers and filled to confirm the closure is water tight. A closed-suction drain is placed and the abdominal incision is closed.

The open surgical approach for urachal adenocarcinoma proceeds in much the same way as for urothelial carcinoma. However, because the urachus may be involved with tumor anywhere along its course, an en bloc resection of the entire urachus as well as umbilicus is required.

\section{Robotic PC}

When performed from a robotic approach, the patient is placed in low lithotomy position. After insufflation of the abdomen, the camera port is placed $2 \mathrm{~cm}$ above the umbilicus. Additionally, flanking paramedian robotic instrument ports and a far left robotic instrument port are placed. A $12 \mathrm{~mm}$ air seal port is placed in the right flank. This is the same configuration as is utilized in a robotic RC (18). Once the ports are placed, a simultaneous flexibly cystoscopy is performed using $\mathrm{CO}_{2}$ (pneumovesicum). By using pneumovesicum, instead of saline irrigant, spillage of tumor cells can be prevented once the bladder is opened. Moreover, some will instill mitomycin $\mathrm{C}$ in the bladder prior to docking the robot to decrease the risk of tumor seeding (19). Using dual vision screens and the light from the cystoscopy as a guide, the surgeon at console can score the bladder with a monopolar scissor (20). It should be mentioned that the light source can be difficult to see with robot assisted laparoscope due to washout from the intraabdominal light source. To avoid this, the light from the laparoscope can be dimmed. The cystoscope can then be removed or remain in until an incision is made into the healthy part of the bladder, at which time a urethral catheter is placed. Whenever possible, the bladder is left attached to the anterior abdominal wall to maximize visualization (21). However, some cases may require mobilization to find a safe area to make the cystotomy. The tumor and $1-2 \mathrm{~cm}$ surrounding bladder margin is then excised using monopolar scissors. An alternative to the scissors for excision of the tumor while using the robot is the EndoWrist One ${ }^{\mathrm{TM}}$ Vessel Sealer. During the excision, the indwelling foley catheter can be intermittently attached to suction to avoid spillage. The specimen is then immediately placed in the specimen bag for later removal. The bladder defect is closed in 2 layers with barbed suture and a bilateral pelvic lymphadenectomy is performed (22).

In order to prevent positive surgical margins, better identification of the tumor edges is required. Kim et al. incorporated technology first used in laparoscopic gastrointestinal surgery of polyps and colon cancer (23). They used endoscopic India ink inside a Deflux needle to tattoo a $1 \mathrm{~cm}$ outer margin around the bladder tumor. Then, they performed a robotic PC, opening the bladder, identifying the tattooed margins, and excising the tumor. Hockenberry et al. used near-infrared fluorescence through the robotic lens without an injectable contrast agent (24). By switching to fluorescence imaging, the white light from the cystoscope illuminated green, allowing for easier marking of the margins for resection, while still maintaining the surrounding field of vision. Alternatively, intra-tumor injection of indocyanine green (ICG) can be utilized to localize the bladder lesion to facilitate partial bladder resection with negative margins, as reported in abstract form and reviewed by Pathak et al. $(25,26)$. Manny et al. has used fluorochrome, ICG, to identify the sentinel lymph node packets during our pelvic lymph node dissection $(27,28)$. In the study evaluating the efficacy of detecting the sentinel nodes, ICG was cystoscopically injected into the tumor. Then, then during the pelvic lymph node dissection, 
node packets containing fluorescent nodal material by nearinfrared fluorescence were sent for pathologic exam.

\section{Laparoscopic PC}

Similar to that of a laparoscopic RC, the patient is placed in lithotomy position (29). After insufflation of the abdomen, a camera port is placed $2 \mathrm{~cm}$ above the umbilicus. Two 12-mm flanking, paramedian ports are then placed, followed by a fourth and fifth $5 \mathrm{~mm}$ port $2.5 \mathrm{~cm}$ anterior and medial to the anterior superior iliac spine (30). The remainder of the operation proceeds in much the same way as previously described using the robotic approach.

A complete resection can be more challenging from a laparoscopic approach. This holds especially true for urachal tumors, as the port configuration must be able to access the entire length of the urachus. As a result, Wadhwa et al. described their laparoscopic technique specific to urachal tumors, with modifications based on tumor size (21). In their description, the anatomic boundaries of resection included resection of the tumor, resection of the peritoneum lateral to the medial umbilical ligaments, the posterior sheath of the rectus muscle of the abdomen to the arcuate line and the muscle fibers of the rectus muscle below it, the extraperitoneal fat in the space of Retzius, and the urachus up to the umbilicus superiorly, sparing the umbilical skin. Ports are placed in the standard fashion, with the camera port $3 \mathrm{~cm}$ cephalad to the umbilicus. For tumors less than $5 \mathrm{~cm}$, an antegrade approach is taken. The anterior abdominal wall peritoneum is incised lateral to the medial umbilical ligament on either side, at the level of the inguinal canal and directed cephalad toward the umbilicus. The lateral incisions meet in the midline at the level of the umbilicus. The urachus is then disconnected from the umbilical skin and placed on tension to allow dissection of the entire urachus with the overlying peritoneum, keeping to the previously mentioned boundaries of dissection. This dissection is carried caudally, entering the space of Retzius, thus mobilizing the bladder off the anterior abdominal wall. A cystotomy is made $1.5 \mathrm{~cm}$ away from where the tumor is visualized, allowing for inspection of the inner surface of the bladder. The tumor is resected en bloc and the bladder is repaired in standard fashion. For tumors greater than $5 \mathrm{~cm}$, a retrograde approach is utilized. With this approach, they began as before, making the lateral peritoneal incisions. However, instead of proceeding antegrade with urachal mobilization, they instead developed the space of Retzius while keeping the tumor attached to the anterior abdominal wall. A posterior wall cystotomy was made to visualize the tumor, The tumor was subsequently resected, placed in an Endocatch bag, and then placed on tension to aid in dissecting out the urachus in a retrograde fashion towards the umbilicus.

\section{Oncologic outcomes}

There are no randomized controlled trials comparing PC to RC. However, a number of retrospective studies and case series suggest that in the appropriately selected patients, a PC may be an acceptable alternative (Table 1). Series out of Memorial Sloan Kettering and MD Anderson both suggested adequate outcomes over a decade ago, which in effect renewed interest in the PC $(6,9)$.

In Holzbeierlein et al., 115 patients underwent PC, of which 58 were for primary bladder tumors of nonurachal origin. Their cohort consisted of predominantly clinical stage T2 unifocal disease without CIS. Of note, there were patients with CIS and multifocal disease. The majority of patients received neoadjuvant chemotherapy as part of a bladder sparing protocol. At a mean follow-up of 33.4 months, 40 patients (69\%) had no evidence of disease. There was a local recurrence in 11 patients (19\%). Seven patients had superficial disease managed with TURBT. An RC was attempted in the four with locally advanced disease, but two were considered unresectable at time of surgery. Twelve patients $(21 \%)$ developed metastatic disease after PC. There was a $10 \%$ complication rate with no perioperative deaths and a median hospital stay of 6 days. At 5 years, 43 patients (74\%) are alive with an intact bladder. Most importantly, this group evaluated the various risk factors for disease recurrence, and provided recommendations for selecting appropriate patients.

Kassouf et al. examined 37 patients undergoing PC, of which none had CIS or multifocal disease. At a mean follow up of 72.6 months, 19 patients $(51 \%)$ were disease free. Nine patients (24\%) had a superficial recurrence that was managed with transurethral resection. Nine patients (24\%) had advanced recurrence of either muscle-invasive disease or metastasis. 18 patients (49\%) had an intact bladder with no disease after 62 months. Calculated 5-year overall survival (OS), disease-specific survival (DSS), and recurrence-free survival were $67 \%, 87 \%$, and $39 \%$.

Another group reported on their findings shortly after these two series were published. Smaldone $e t$ al. analyzed data on 25 patients with either T2 or HGT1 unifocal disease without CIS (10). As part of their protocol, they gave all patients preoperative radiotherapy localized to the abdominal wall, a single intraoperative dose of intravesical thiotepa before tumor excision, and 6 weeks of intravesical 
Table 1 Summary of retrospective reviews and case series evaluating oncologic efficacy of partial cystectomy

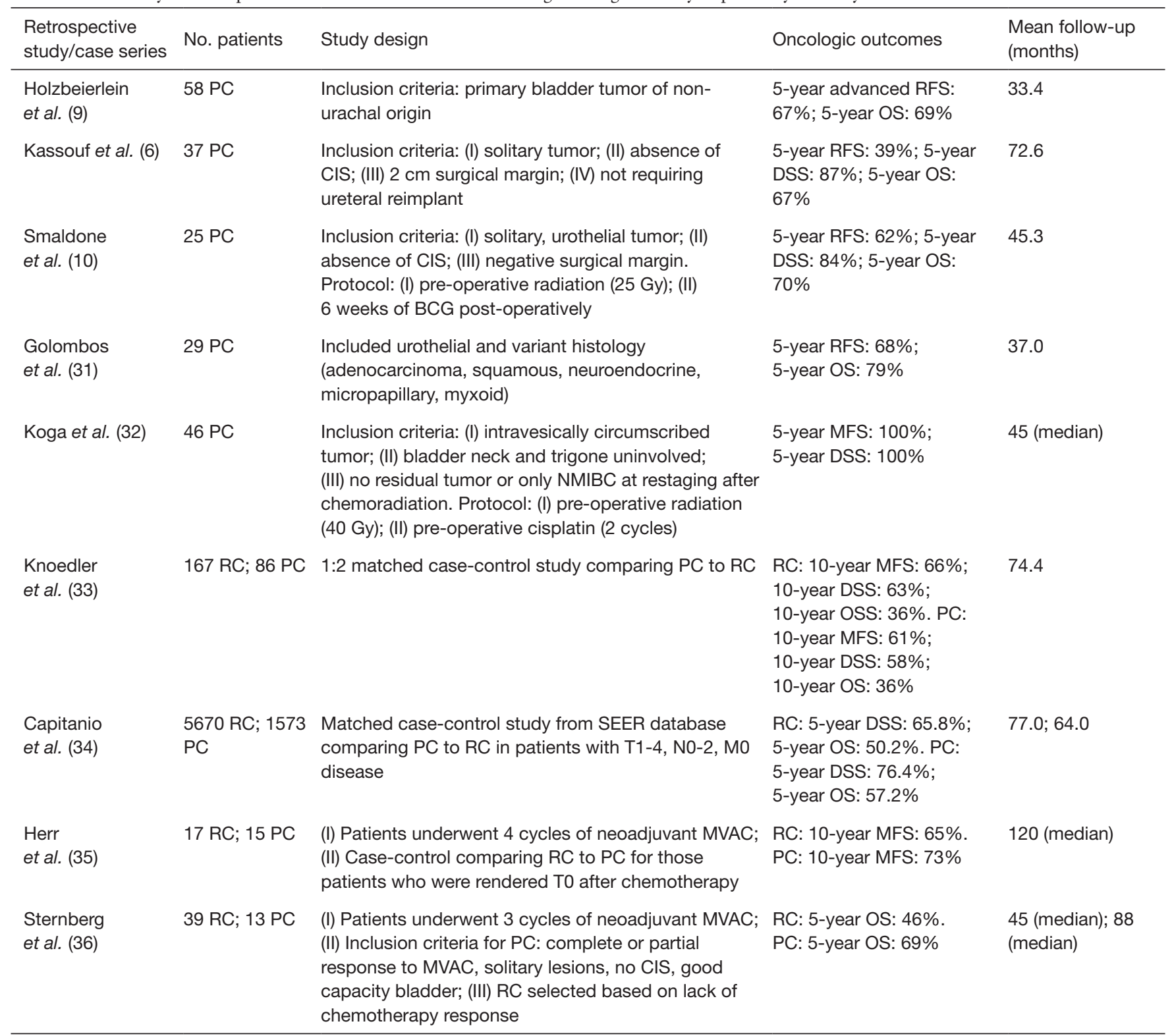

PC, partial cystectomy; RC, radical cystectomy; RFS, recurrence-free survival; MFS, metastasis-free survival; DSS, disease-specific survival; OS, overall survival; NMIBC, non-muscle invasive bladder cancer; MVAC, methotrexate, vincristine, doxorubicin, cisplatin.

BCG therapy postoperatively. Sixty-four percent of patients received a pelvic lymphadenectomy. With mean follow up of 45.3 months, 5 -year recurrence-free, disease-specific, and overall survival rates were $62 \%, 84 \%$, and $70 \%$, respectively. Three patients (12\%) had non-muscle-invasive recurrence, two patients $(8 \%)$ had muscle invasive recurrence, two patients (8\%) had locally advanced recurrence, and one patient (4\%) had metastatic recurrence. There were two major perioperative complications, but no perioperative deaths.
The largest robotic PC series was recently published by Golombos et al. (31). This group identified 29 patients who underwent robotic PC as definitive management of a primary bladder tumor. With a median follow-up of 37 months, the 5 -year OS and RFS rates were $79 \%$ and $68 \%$, respectively. Seven patients (24\%) had a recurrence, three of which were local and four of which were regional or distant. Two local recurrences were managed with TURBT because there was no muscle invasion. The third local recurrence was 
muscle-invasive, and thus managed with a cystectomy.

Using the most stringent criteria, Koga et al. reported on selective bladder-sparing protocol (32). Patients underwent a debulking TURBT with random biopsy, and were subsequently considered for PC if they had intravesically circumscribed tumors $\leq 25 \%$ of the bladder area and no involvement of the bladder neck or trigone. They then underwent radiation and two cycles of cisplatin, before being restaged 4-6 weeks later. To qualify as a PC candidate, the restaging TURBT must show no evidence of disease or small amounts of non-muscle invasive disease. Of their initial cohort of 183 patients, 65 met criteria, of which 46 actually underwent PC. Seven patients developed intravesical recurrence, all of which was superficial. Fiveyear CSS for this cohort was $100 \%$.

Knoedler et al. performed a matched-control analysis comparing PC to RC (33). Patients undergoing partial PC, like the previously mentioned studies, had a single bladder tumor without associated CIS. In this analysis, $167 \mathrm{RC}$ patients were matched in a 2:1 fashion to $86 \mathrm{PC}$ patients. They were matched based on age, gender, pathological stage and receipt of neoadjuvant chemotherapy. It was unclear whether they were originally matched based on multifocality and absence of CIS. And as expected on analysis, patients undergoing PC were less likely to have multifocal bladder tumors on final pathology as compared to RC (15.1\% vs. $32.9 \%$ ). $38 \%$ of patients who underwent a PC developed an intravesical recurrence. Five percent of PC patients experienced a pelvic recurrence. At a mean follow-up of 6.2 years, $81 \%$ of patients maintained an intact bladder. Most significant, no differences existed between PC and $\mathrm{RC}$ with regard to 10 -year metastasis free survival $(61 \%$ vs. $66 \%$ ), cancer-specific survival (CSS) (58\% vs. $63 \%)$, or overall survival (36\% vs. 36\%).

A second study examined the SEER database, comparing overall survival and cause-specific survival in patients treated with PC versus those treated with RC (34). In this analysis, Capitanio et al. performed three separate analyses. The first was of the entire cohort of 7,243 who had pT1-4N1-2M0 disease and underwent either PC or $\mathrm{RC}$. They repeated the comparison between PC and RC after matching in a 1:4 ratio for tumor grade, pT stage, $\mathrm{pN}$ stage, age, race, and year of surgery. A second matched analysis was performed after adding the number of removed lymph nodes to the matching criteria. In the first analysis, the OS and CSS estimates at five years were $57.2 \%$ and $76.4 \%$ for PC patients, respectively, and $50.2 \%$ and $65.8 \%$ for $\mathrm{RC}$ patients, respectively $(\mathrm{P}<0.001)$. In the second analysis, the OS and CSS estimates were $56.0 \%$ and $73.5 \%$ for PC patients, respectively, and $50.9 \%$ and $67.5 \%$ for $\mathrm{RC}$ patients, respectively ( $\mathrm{P}=0.03$; and $\mathrm{P}<0.001$ for both). When the number of removed lymph nodes was included in the third analysis, the 5-year OS and CSS estimates were $57.2 \%$ and $70.3 \%$, respectively, for PC patients and $54.6 \%$ and $69.2 \%$, respectively, for RC patients.

With regard to the application of neoadjuvant chemotherapy, there is little literature to guide management. Herr et al. reported on a group of 60 patients who achieved cT0 after receiving MVAC chemotherapy followed by TURBT (35). Fifteen of these patients subsequently underwent PC and 17 underwent RC. The 10-year metastasis free survival was $73 \%$ for those who underwent PC, and $65 \%$ for those who underwent RC. Furthermore, $53 \%$ of patients in the PC group had intact bladders. Sternberg et al. performed a similar analysis, evaluating 104 patients with muscle invasive disease who underwent TURBT after MVAC chemotherapy (36). Thirteen patients with history of a solitary lesion and either a complete or partial response underwent PC. Thirty-nine patients underwent RC. Five-year survival for PC was $69 \%$ compared to $46 \%$ for RC.

Many of these series report on recurrences after PC. They are often categorized as either intravesical or extravesical (metastatic). Intravesical disease can further be classified as either superficial, non-invasive or invasive/ locally advanced. Much of the literature reports use of transurethral resection in those patients who presents with recurrent superficial disease. However, a salvage cystectomy should be recommended in those with muscleinvasive or locally advanced disease. A small group of patients will also present with disease having metastasized. These patients often had either positive lymph nodes from surgery, or micrometastatic disease at time of presentation. While it seems logical to offer RC after muscle-invasive recurrence, there is no data to provide guidance on the best treatment for superficial disease. The question of whether these patients would benefit from RC rather than repeat resections remains unanswered.

\section{Complications}

Of the published reports discussed, only three provide the associated complication rates. Holzbeierlein et al. reported 6 complications $(10 \%)$, of which only one would be considered a major complication (9). In Smaldone et al., there were two major complications requiring intervention ( $8 \%)$ out of the 25 patients treated with PC (10). Specifically, one patient required re-exploration, and another required percutaneous drainage of lymphocele. 
Golombos et al. reported two perioperative complications $(6.9 \%)$ and $5(17.9 \%)$ complications within 90 days of surgery, all of which were minor (31). Nevertheless, a comparison of reported complications after $\mathrm{RC}$ reveals that the morbidity associated with a PC is notably less. Indeed, a review by Shabsigh et al. found that of 1,142 patients undergoing $\mathrm{RC}$, there was a $64 \%$ total complication rate and $13 \%$ major complication (3). Therefore, offering PC in an appropriately selected patient who does not accept or cannot tolerate the associated morbidity of an RC, represents an acceptable alternative.

\section{Conclusions}

PC with a bilateral pelvic lymph node dissection can be offered to the appropriately selected surgical candidate. While only $5-10 \%$ of patients with muscle invasive bladder cancer would be considered good candidates, it remains underutilized as many are concerned with compromising oncologic success. More recent literature has demonstrated that in the short-term, acceptable oncologic outcomes are achievable. Clinicians must keep in mind, however, that this is an alternate approach. The most definitive and durable, longterm outcomes remain with RC. As the literature is comprised of only case series and retrospective studies, there Level 1 evidence is lacking in this respect. Additional work must be done to further elucidate the most appropriate candidates, as well as the roles and timing of chemotherapy and radiation in a more comprehensive bladder sparing approach.

\section{Acknowledgments}

Funding: None.

\section{Footnote}

Provenance and Peer Review: This article was commissioned by the Guest Editors (Ja Hyeon Ku, Ho Kyung Seo, Seok Ho Kang) for the series "Muscle-Invasive Bladder Cancer" published in Translational Andrology and Urology. The article has undergone external peer review.

Conflicts of Interest: Both authors have completed the ICMJE uniform disclosure form (available at http://dx.doi. org/10.21037/tau.2020.03.04). The series "Muscle-Invasive Bladder Cancer" was commissioned by the editorial office without any funding or sponsorship. Dr. Hemal serves as an unpaid editorial board member of Translational Andrology and Urology from May 2019 to May 2021. The authors have no other conflicts of interest to declare.
Ethical Statement: The authors are accountable for all aspects of the work in ensuring that questions related to the accuracy or integrity of any part of the work are appropriately investigated and resolved.

Open Access Statement: This is an Open Access article distributed in accordance with the Creative Commons Attribution-NonCommercial-NoDerivs 4.0 International License (CC BY-NC-ND 4.0), which permits the noncommercial replication and distribution of the article with the strict proviso that no changes or edits are made and the original work is properly cited (including links to both the formal publication through the relevant DOI and the license). See: https://creativecommons.org/licenses/by-nc-nd/4.0/.

\section{References}

1. Siegel RL, Miller KD, Jemal A. Cancer statistics, 2019. CA Cancer J Clin 2019;69:7-34.

2. Djaladat H, Katebian B, Bazargani ST, et al. 90Day complication rate in patients undergoing radical cystectomy with enhanced recovery protocol: a prospective cohort study. World J Urol 2017;35:907-11.

3. Shabsigh A, Korets R, Vora KC, et al. Defining early morbidity of radical cystectomy for patients with bladder cancer using a standardized reporting methodology. Eur Urol 2009;55:164-74.

4. Yuh B, Torrey RR, Ruel NH, et al. Intermediate-term oncologic outcomes of robot-assisted radical cystectomy for urothelial carcinoma. J Endourol 2014;28:939-45.

5. Kader AK, Richards KA, Krane LS, et al. Robot-assisted laparoscopic vs open radical cystectomy: comparison of complications and perioperative oncological outcomes in 200 patients. BJU Int 2013;112:E290-4.

6. Kassouf W, Swanson D, Kamat AM, et al. Partial cystectomy for muscle invasive urothelial carcinoma of the bladder: a contemporary review of the M. D. Anderson Cancer Center experience. J Urol 2006;175:2058-62.

7. Gray PJ, Fedewa SA, Shipley WU, et al. Use of potentially curative therapies for muscle-invasive bladder cancer in the United States: results from the National Cancer Data Base. Eur Urol 2013;63:823-9.

8. Fedeli U, Fedewa SA, Ward EM. Treatment of muscle invasive bladder cancer: evidence from the National Cancer Database, 2003 to 2007. J Urol 2011;185:72-8.

9. Holzbeierlein JM, Lopez-Corona E, Bochner BH, et al. Partial cystectomy: a contemporary review of the Memorial Sloan-Kettering Cancer Center experience and recommendations for patient selection. J Urol 2004;172:878-81. 
10. Smaldone MC, Jacobs BL, Smaldone AM, et al. Longterm results of selective partial cystectomy for invasive urothelial bladder carcinoma. Urology 2008;72:613-6.

11. Peress JA, Waterhouse K, Cole AT. Complications of partial cystectomy in patients with high grade bladder carcinoma. J Urol 1977;118:761.

12. Fox M, Power RF, Bruce AW. Diverticulum of the bladder-presentation and evaluation of treatment of 115 cases. $\mathrm{Br}$ J Urol 1962;34:286-98.

13. Prakash, Rajini T, Kumar Bhardwaj A, et al. Urinary bladder diverticulum and its association with malignancy: an anatomical study on cadavers. Rom J Morphol Embryol 2010;51:543-5.

14. Siefker-Radtke A. Urachal carcinoma: surgical and chemotherapeutic options. Expert Rev Anticancer Ther 2006;6:1715-21.

15. Johnson DE, Hodge GB, Abdul-Karim FW, et al. Urachal carcinoma. Urology 1985;26:218-21.

16. Kikuno N, Urakami S, Shigeno K, et al. Urachal carcinoma associated with increased carbohydrate antigen 19-9 and carcinoembryonic antigen. J Urol 2001;166:604.

17. Guarnaccia S, Pais V, Grous J, et al. Adenocarcinoma of the urachus associated with elevated levels of CA 125 . J Urol 1991;145:140-1.

18. Pathak RA, Patel M, Hemal AK. Comprehensive Approach to Port Placement Templates for RobotAssisted Laparoscopic Urologic Surgeries. J Endourol 2017;31:1269-76.

19. Bailey GC, Frank I, Tollefson MK, et al. Perioperative outcomes of robot-assisted laparoscopic partial cystectomy. J Robot Surg 2018;12:223-8.

20. Singh I, Kader K, Hemal AK. Robotic distal ureterectomy with reimplantation in malignancy: technical nuances. Can J Urol 2009;16:4671-6.

21. Wadhwa P, Kolla SB, Hemal AK. Laparoscopic en bloc partial cystectomy with bilateral pelvic lymphadenectomy for urachal adenocarcinoma. Urology 2006;67:837-43.

22. Peak TC, Hemal AK. Anatomic Robot-Assisted Radical Cystectomy. Robotics in Genitourinary Surgery 2018:715-32.

23. Kim BK, Song MH, Yang HJ, et al. Use of cystoscopic tattooing in laparoscopic partial cystectomy. Korean J Urol 2012;53:401-4.

24. Hockenberry MS, Smith ZL, Mucksavage P. A novel use of near-infrared fluorescence imaging during robotic surgery without contrast agents. J Endourol 2014;28:509-12.

25. Pathak RA, Hemal AK. Intraoperative ICG-fluorescence imaging for robotic-assisted urologic surgery: current status and review of literature. Int Urol Nephrol 2019;51:765-71.

26. Rodriguez Morales-Bermudez AR. 980 Fluorescence imaging technology for robotic assisted partial cystectomy and ureteral reconstruction minimizes lack of tactile feedback. European Urology Supplements 2015;14:e980.

27. Manny TB, Hemal AK. Fluorescence-enhanced robotic radical cystectomy using unconjugated indocyanine green for pelvic lymphangiography, tumor marking, and mesenteric angiography: the initial clinical experience. Urology 2014;83:824-9.

28. Patel MN, Hemal AK. Molecular Targeted FluorescenceGuided Intraoperative Imaging of Bladder Cancer Nodal Drainage Using Indocyanine Green During Radical and Partial Cystectomy. Curr Urol Rep 2016;17:74.

29. Hemal AK, Singh I, Kumar R. Laparoscopic radical cystectomy and ileal conduit reconstruction: preliminary experience. J Endourol 2003;17:911-6.

30. Ansari MS, Hemal AK. A rare case of urachovesical calculus: a diagnostic dilemma and endo-laparoscopic management. J Laparoendosc Adv Surg Tech A 2002;12:281-3.

31. Golombos DM, O'Malley P, Lewicki P, et al. Robotassisted partial cystectomy: perioperative outcomes and early oncological efficacy. BJU Int 2017;119:128-34.

32. Koga F, Kihara K, Yoshida S, et al. Selective bladdersparing protocol consisting of induction low-dose chemoradiotherapy plus partial cystectomy with pelvic lymph node dissection against muscle-invasive bladder cancer: oncological outcomes of the initial 46 patients. BJU Int 2012;109:860-6.

33. Knoedler JJ, Boorjian SA, Kim SP, et al. Does partial cystectomy compromise oncologic outcomes for patients with bladder cancer compared to radical cystectomy? A matched case-control analysis. J Urol 2012;188:1115-9.

34. Capitanio U, Isbarn H, Shariat SF, et al. Partial cystectomy does not undermine cancer control in appropriately selected patients with urothelial carcinoma of the bladder: a population-based matched analysist. Urology 2009;74:858-64.

35. Herr HW, Bajorin DF, Scher HI. Neoadjuvant chemotherapy and bladder-sparing surgery for invasive bladder cancer: ten-year outcome. J Clin Oncol 1998;16:1298-301.

36. Sternberg CN, Pansadoro V, Calabro F, et al. Can patient selection for bladder preservation be based on response to chemotherapy? Cancer 2003;97:1644-52.

Cite this article as: Peak TC, Hemal A. Partial cystectomy for muscle-invasive bladder cancer: a review of the literature. Transl Androl Urol 2020;9(6):2938-2945. doi: 10.21037/tau.2020.03.04 